\title{
CONDICIONES GENERALES DE LOS ESTUDIANTES DE MEDICINA DE LA GENERACIÓN 2010 DURANTE SU INTERNADO ROTATORIO DE PREGRADO EN LA ESCUELA SUPERIOR DE MEDICINA DEL INSTITUTO POLITÉCNICO NACIONAL
}

\begin{abstract}
Ricardo Fuentes Unzueta ${ }^{1}$, Carolina Manrique Nava ${ }^{1}$, Octaviano Domínguez Márquez ${ }^{1}$
Resumen: En la Escuela Superior de Medicina del Instituto Politécnico Nacional, el Internado se cursa antes del servicio social y consiste en un año de formación dentro del hospital, frente a pacientes, bajo supervisión directa de médicos. Se realizó un estudio descriptivo a la generación de estudiantes de 2010, a quienes se aplicó una encuesta al término de su Internado en 2010. El 57\% fueron mujeres; al 71\% las autoridades no lo respetaron durante su horario de comida; al $60 \%$ los profesores no lo respetaron en su horario de clases; el 25\% fue objeto de hostigamiento; el 35\% objeto de violencia y maltrato, y el 35\% víctima de discriminación. El 61\% se percató de que personal del hospital se alcoholiza o usa estupefacientes dentro del hospital en horas de servicio. Para el 58\%, las autoridades de la escuela no dieron seguimiento a su estancia y el 15\% fue víctima de acoso sexual por médicos de base, residentes y personal de enfermería. Es importante señalar que este es un estudio inicial y único en su tipo. Se realizarán estudios en las siguientes generaciones para encontrar patrones de incidencia o prevalencias significativas, denunciarlas y evitar que se repitan en el futuro.
\end{abstract}

Palabras clave: Internado Rotatorio de Pregrado, pregrado, internos, estudiantes de medicina, bioética médica, ética médica, calidad educativa

General conditions of medicine students, class 2010, during their pre-degree rotatory internship of Medical School, National Polytechnic Institute

\begin{abstract}
In the School of Medicine of the National Polytechnic Institute, internship takes place before social service and consists of a training year inside the Hospital, in which trainees see patients under direct supervision of physicians. A descriptive study was carried out of student's class 2010, to whom a survey was applied by the end of their internship in 2010 . $57 \%$ of students were women; for $71 \%$, lunch time was not respected by authorities; for $60 \%$, schedules of classes were not respected by professors; $25 \%$ students were harassed; $35 \%$ students were object of violence and maltreatment; and $35 \%$ students were victim of discrimination. $61 \%$ students realized that Hospital staff consumes alcohol or drugs inside the Hospital in service hours. For $58 \%$ students, School authorities did not monitor their stay and $15 \%$ students were victim of sexual harassment by physicians, residents and nurse staff. It is important to point out that this is an initial study unique in its type. More studies will be carried out in the following generations to find out incidence patterns or significant prevalence, in order to denounce them and to avoid their future repetition.
\end{abstract}

Key words: Pre-degree rotatory internship, pre-degree, internship, medicine students, medical bioethics, medical ethics, quality training

\section{Condiçóes gerais dos estudantes de medicina da turma 2010 durante o seu Internato Rotatório de Graduaçáo na} Escola Superior de Medicina do Instituto Politécnico Nacional

Resumo: Na Escola Superior de Medicina do Instituto Politécnico Nacional, o Internato é cursado antes do servicio social e consiste em um ano de formaçáo dentro do hospital, junto a pacientes, sob supervisão direta de médicos. Foi realizado um estudo descritivo na turma de estudantes de 2010, aos quais foi aplicada uma enquete no término de seu Internato em 2010. $57 \%$ eram mulheres; para $71 \%$ as autoridades não respeitaram seu horário de refeição; para $60 \%$ os professores não respeitaram o seu horário de aulas; $25 \%$ foram objeto de hostilização; $35 \%$ foram objeto de violência e maltrato, e 35\% foram vítimas de discriminação. $61 \%$ se conscientizaram de que o pessoal do hospital se alcooliza ou usa estupefacientes dentro do hospital no horário de serviço. Para 58\% as autoridades da escola não deram seguimiento ao seu estágio e $15 \%$ foram vítimas de assédio sexual por médicos de base, residentes e pessoal de enfermagem. É importante assinalar que este é um estudo inicial e único de sua categoria. Foram realizados estudos nas turmas seguintes para encontrar padróes de incidência ou prevalências significativas, denunciá-las e evitar que se repitam no futuro.

Palavras-chave: Internato Rotatório de Graduação, graduação, internos, estudantes de medicina, bioética médica, ética médica, qualidade educativa

\footnotetext{
${ }^{1}$ Escuela Superior de Medicina del Instituto Politécnico Nacional (IPN), México

Correspondencia: ryckworld@hotmail.com
} 


\section{Introducción}

Se conoce muy poco sobre la formación del personal de salud en nuestro país. La medicina es una de las disciplinas más humanas por definición. Con la aparición de la bioética se han desarrollado estudios para cuidar que la ética y el humanismo no se pierdan. La denuncia del paternalismo en la relación médico-paciente, la defensa de los derechos del paciente y el consentimiento con información han surgido para evitar abusos u omisiones del personal de salud(1).

En México un tema de dominio público y evidente para toda persona es el pésimo servicio del sistema público de salud. Los hospitales públicos más grandes e importantes del país son también famosos por sus malos tratos y negligencias médicas.

Toda persona que ha ingresado a alguna unidad de este tipo ha podido presenciar y ser sujeto (por no decir objeto) de muchas prácticas y acciones contrarias al respeto de la dignidad, los derechos humanos, o a un trato humano y respetuoso(2).

La mayoría de los estudiantes de medicina esperan ansiosamente el momento para estar frente al paciente y cumplir con su deseo más grande (o lo que se esperaríamos que fuera su deseo más grande), a saber, ayudar, preservar y mejorar la salud de las personas. Por lo anterior, el Internado debería ser la etapa más positiva, bella e inolvidable de todas.

No todo es fácil en su formación. Al ingresar al hospital se enfrentan cada día a la muerte, al sufrimiento y a la fragilidad humana. Sin embargo, con cada una de sus acciones pueden mejorar el estado en que se encuentran las personas.

Los estudiantes ingresan para aprender la práctica médica no en la teoría sino en la práctica y en la realidad del hospital. Los responsables de su formación en esta etapa son los médicos adscritos, quienes cada año reciben a las nuevas generaciones(3).

El Internado consiste en un año de formación frente a pacientes en unidades de salud de $2^{\circ} \mathrm{o}$ $3^{\text {er }}$ nivel de atención. El marco jurídico en el que se encuentran las obligaciones, características, duración, actividades, responsables y sanciones es NOM-234-SSA1-2003(4), utilización de campos clínicos para ciclos clínicos e internado de pregrado.

La presente investigación surge con el fin de conocer las características y condiciones a las que se enfrentan los estudiantes de medicina durante este año de formación dentro del hospital. El Internado es una etapa clave, porque es el primer momento en que empiezan a formar parte del equipo de salud en los hospitales más importantes del país. Sus modelos y maestros son los médicos de base y especialistas que conocen en los diferentes servicios; sin embargo, ¿cómo es la vida dentro del hospital? ¿A qué se enfrentan? ¿Cómo los tratan y cuáles son sus condiciones de vida en esta etapa?

No existen investigaciones que muestren cómo es la vida de los médicos a lo largo de su formación. Este estudio utiliza, por analogía, las mismas preguntas exploratorias que el médico hace al paciente para darse una idea del mal que le aqueja desde su perspectiva. El siguiente paso es la auscultación minuciosa del paciente (registro de signos vitales, talla, peso y toda la historia clínica) para, una vez detectadas algunas posibles causas, hacer los estudios y radiografías correspondientes con el fin de conocer realmente la magnitud y origen del problema. Los estudios posteriores serán los primeros diagnósticos para confirmar qué es verdad y qué no. Es importante el seguimiento y desarrollo de este tipo de investigaciones en cada unidad y país para conocer en profundidad las condiciones de la educación médica en el mundo. Con investigaciones serias y duraderas se podrá conocer si el problema es solo un raspón, si es una fractura o si habrá que amputar alguna parte. Lo que está en juego es la formación y vida de nuestros jóvenes médicos encargados de la atención de la salud a toda la población.

\section{Método}

Se realizaron entrevistas a profundidad a cinco médicas que habían terminado su Internado, para conocer con detalle las condiciones y situaciones que vivieron. Posteriormente, con las preguntas más relevantes, se desarrolló un instrumento. Se 
realizó una prueba piloto a un grupo de 37 médicos de diferentes generaciones que habían terminado su Internado, para conocer sus comentarios y retroalimentación de las preguntas. Finalmente, con las aportaciones previas, se rediseño el instrumento y se aplicó a 135 alumnos de la generación 2010, al término de su Internado. Finalmente, se analizaron los datos con el programa SPSS, versión 2.0.

\section{Resultados}

\section{Población}

El $41 \%$ son hombres y el $57 \%$ mujeres; de ellos, el $85 \%$ tiene entre 23 y 26 años.

\section{Instalaciones}

El 34\% no contó con instalaciones para su aseo personal. El 28\% no contó con áreas de descanso. De los estudiantes que sí contaron con instalaciones, el $53 \%$ no pudo usarlas, ya que el resto del personal del hospital las usaba.

\section{Guardias de castigo}

Al 27\% de internos lo obligaron a cubrir una o más guardias de castigo; las causas principales fueron llegar tarde, no asistir o comer.

\section{Gráfico 1. Atención médica}

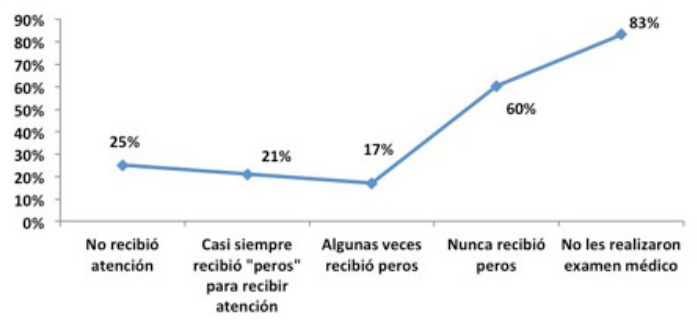

Gráfico 2. Porcentaje de internos atendiendo el servicio solos y realizando procedimientos sin instrucción

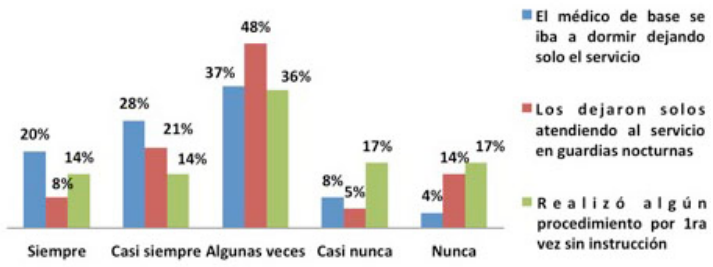

\section{Ética y humanismo}

Para el 35\%, el trabajo del personal del hospital no promovió en ellos una práctica ética y humanista fruto de su ejemplo. Para el 17\%, el coordinador de internos nunca cuidó que la ética, moral y disciplina se mantuviera y no afectara la integridad del personal; para el $30 \%$ algunas veces y para el 51\% casi siempre.

Para el 72\%, el Internado casi siempre ayudó a fortalecer un espíritu ético y humanista. Al 71\% no le respetaron sus horario de comida. El 50\% tuvo dos comidas o menos y el $47 \%$ tres o más de tres comidas.

$\mathrm{Al} 34 \%$ algunas veces le hicieron propuestas de recibir enseñanza a cambio de algo, y al $6 \%$ casi siempre. Las propuestas más frecuentes fueron dinero, comida y sexo.

Al 50\% los médicos adscritos casi siempre le resolvieron sus dudas y al $45 \%$ algunas veces. Según el 61\%, el personal calificado casi siempre rectificó sus acciones; al 35\% algunas veces al resto casi nunca o nunca.

Para el 68\%, el Internado casi siempre le ofreció una educación acorde con las necesidades actuales. Para el 64\% la información casi siempre estuvo actualizada y solo el $37 \%$ consideró que al docente le importó su aprovechamiento.

Gráfico 3. Actividades acorde a su formación

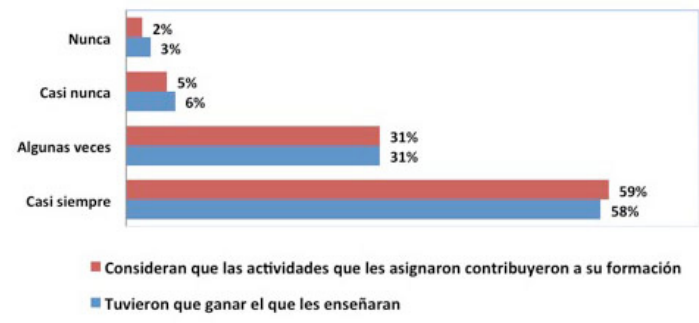

\section{Clases y conocimientos}

El $76 \%$ casi siempre aplicó sus conocimientos previos durante el Internado, el 22\% algunas veces y el 2\% nunca. El 48\% de las veces los internos dieron las clases y el $43 \%$ las dieron el maestro y ellos.

Al 27\% el Internado no lo dotó de las competen- 
cias necesarias para ofrecer una atención de calidad y para el 16\% la evaluación teórica no correspondió con lo expuesto en clase. Para el $64 \%$ las calificaciones no reflejaron su aprovechamiento y al $60 \%$ los profesores no lo respetaron durante su horario de clase.

Gráfico 4. Carga de trabajo

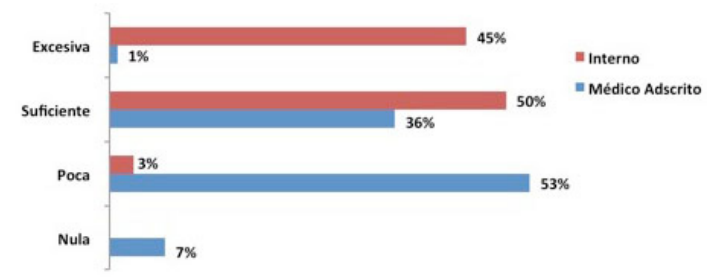

Para el 13\% el Internado no fue una experiencia positiva para su desarrollo académico y formativo

Gráfico 5. Jerarquías

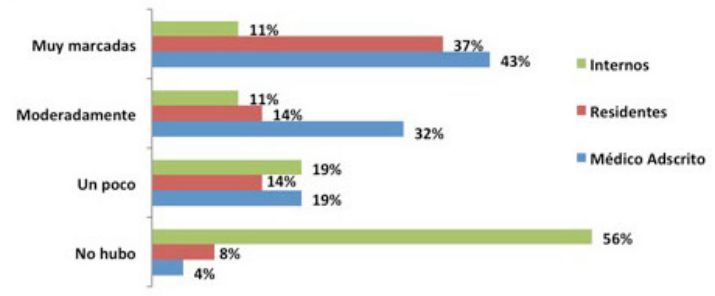

Acoso Sexual

El 15\% sufrió acoso sexual y un 5\% no contestó la pregunta. Solo seis lo denunciaron y dos de ellos sufrieron represalias por hacer la denuncia.

Gráfico 6. Hostigamiento, maltrato y discriminación

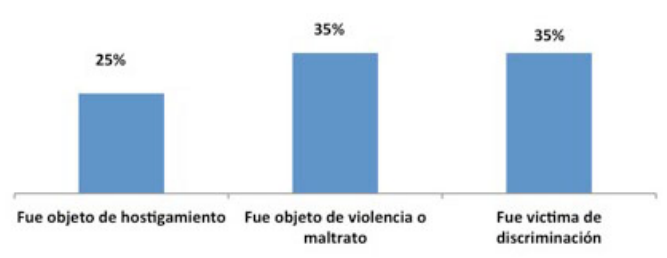

Gráfico 7. Consumo de alcohol o drogas

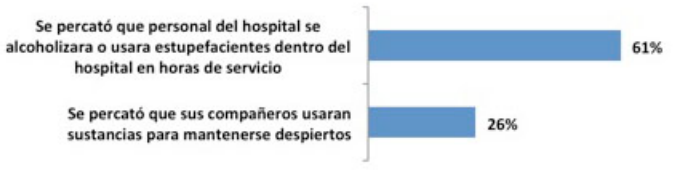

Gráfico 8. Contacto con la familia y actividades recreativas

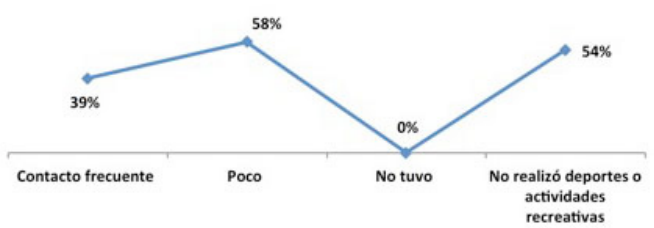

Seguimiento de autoridades

El 58\% respondió que las autoridades de la Escuela Superior de Medicina no dieron seguimiento a su estancia durante su Internado y el 63\% que la información del curso de inducción realizado por estas autoridades no correspondió con lo que vivieron durante el Internado.

El 67\% de los internos contesto que el Internado les ayudó a fomentar una cultura de salud física y mental.

\section{Discusión}

Los datos presentados son el resultado de un estudio inicial que muestra la realidad de la práctica médica para estos estudiantes durante su Internado.

La relevancia del tema radica en que estos estudiantes ingresan a los hospitales más importantes del país. La mayoría de estas unidades se encuentra en la capital, por lo que no hay justificación por parte de las autoridades, tanto hospitalarias, de salud o educativas, para decir que era imposible evaluar o dar seguimiento a los estudiantes durante esta etapa. 
Si bien los resultados no pueden tomarse como absolutos sirven para a) tener un panorama más general y más real de lo que pasa en los hospitales, y b) tener una noción más completa de cómo es la formación de los estudiantes durante esta etapa.

Aunque el estudio muestra los resultados de la población de una universidad, es casi un hecho que las percepciones y respuestas de los estudiantes de otras universidades serán como estas, ya que todos pasan por los mismos hospitales y doctores. La realidad del hospital es la misma para todos.

Los resultados son preocupantes y requieren de un seguimiento y estudio a las siguientes generaciones para identificar las prevalencias más importantes y graves que atentan contra la adecuada formación de los estudiantes.

Todo el personal de salud es un mismo equipo y todos sus integrantes son igualmente importantes y fundamentales para la relación médico-paciente y para el adecuado funcionamiento de cada unidad. Por ello es fundamental conocer cuáles son las condiciones en las que se encuentran dentro del hospital, cómo es su vida durante su formación y cómo es el trato entre ellos. Todas estas investigaciones aportarán datos fundamentales para entender al sistema de salud en México y cómo se transmiten estos modelos y mala praxis generación tras generación.

No se debe olvidar que es responsabilidad de las autoridades estar informadas y conocer en detalle las condiciones en que se encuentran estudiantes, trabajadores y pacientes. Los datos aquí presentados muestran deficiencias profundas respecto del cumplimiento de sus obligaciones con los estudiantes de pregrado.

Los documentos más importantes en los que se encuentran los objetivos, actividades y condiciones sobre el Internado son la Norma Oficial Mexicana (NOM) y el Programa Académico de la Escuela Superior de Medicina.

\section{Instalaciones}

La NOM-234-SSA1-2003, en su artículo 5.1, menciona que los establecimientos para la atención médica deben contar con áreas de descanso, áreas de aseo personal exclusivas para internos y comedor. Sin embargo, en la realidad no todos los estudiantes cuentan con las instalaciones; además, un porcentaje importante de internos no puede ocupar sus lugares porque el resto del personal del hospital los está ocupando.

La NOM, en su artículo 7.5.4, señala que las prácticas clínicas complementarias o guardias de "castigo" no son aplicables. Están prohibidas; sin embargo, siguen ocurriendo con mucha frecuencia y lo grave es que son los propios médicos adscritos quienes las fomentan y aplican.

Otro tema de gravedad es lo relacionado con la atención del paciente. En los resultados es clara la negligencia y falta de responsabilidad de los médicos adscritos, al dejar solos a los internos atendiendo al paciente. La NOM establece que los profesores deben proporcionar a los alumnos enseñanza tutorial y no exponerlos a actividades sin asesoría o supervisión que impliquen responsabilidad legal. También, en su artículo 5.1, menciona que debe haber un médico responsable del control, supervisión, asesoría y evaluación de los alumnos durante el desarrollo del curso por cada área de rotación. Debe haber además personal médico legalmente responsable de los servicios de atención médica las 24 horas de los 365 días del año. Al igual que la NOM, el Programa Académico menciona que todas las actividades deben realizarse bajo la asesoría del profesor hasta que se tenga certeza de que la aplicación es correcta.

La tarea del médico adscrito es supervisar constantemente el aprendizaje, habilidades y destrezas de los internos a su cargo. Los hallazgos muestran el vacío existente en el corazón del Internado: la formación del interno. El Internado es la etapa formativa práctica de los estudiantes y ellos están ahí para recibir enseñanza y aprender de los adscritos. Sin embargo, como se puede apreciar, se deja a los estudiantes solos, sin instrucción y como responsables de los pacientes.

\section{Etica y humanismo}

Los estudiantes no encuentran en las prácticas del personal de salud una actitud ética o humana. Al preguntarles sobre la actitud del coordinador, la respuesta fue que este no cuida que la ética, la 
moral o la disciplina se mantenga, y no afecte en consecuencia la integridad del personal.

El Internado es, por definición, una etapa formativa, sin embargo, son frecuentes las propuestas a estudiantes de recibir enseñanza a cambio de favores. Lo anterior representa una seria crisis de valores de las autoridades. Son atentados directos contra la responsabilidad, la ética, la dignidad, el profesionalismo y la justicia. Los abusos de autoridad y de poder son evidentes y los alumnos no cuentan con mecanismos para denunciar o evitar este tipo de acciones de sus superiores.

No existe una cultura de denuncia. También es evidente la falta de decisión de los estudiantes para reconocer estos actos y oponerse a ellos. ¿Es verdad que nadie en el hospital se da cuenta de todos estos actos? ¿Qué hace el resto del personal ante estas faltas evidentes contra sus derechos y dignidad? Lo más preocupante es que todos estos resultados y problemas los conocen todos los médicos; ellos, en sus años de formación, vivieron lo mismo y, ahora que son autoridades o encargados de los estudiantes, siguen sin intentar cambiarlo.

Acoso sexual, hostigamiento, violencia y discriminación

Los estudiantes fueron objeto de acoso sexual y alrededor del $30 \%$ fue objeto de hostigamiento, maltrato o discriminación. Un resultado fuera de todo pronóstico fue encontrar que ellos se percataron cuando personal del hospital se alcoholizaba o usaba estupefacientes dentro del hospital en horas de servicio. ¿Por qué pasa eso? ¿Cómo es esto posible?

El 58\% contestó que las autoridades no dieron seguimiento a su estancia. ¿Qué tipo de seguimiento a estudiantes existe al respecto? ¿Qué organismo está encargado de recibir sus denuncias? ¿Cuáles son las denuncias más frecuentes? ¿Cuáles son las unidades o servicios que más se repiten? ¿Fueron reales? ¿Qué se hizo al respecto?

No contamos con información y eso es preocupante: que las autoridades educativas, hospitalarias, de salud y de comités de bioética sigan hablando sobre derechos humanos, dignidad, ética, responsabilidad, justicia y calidad, mientras den- tro de cada unidad los estudiantes son objeto de violaciones a sus derechos.

\section{Jerarquías}

El médico es considerado como una persona con valores, que trata a los demás con respeto e igualdad. Sin embargo, en los resultados sobre jerarquías se encontró información que muestra lo opuesto. Entre el personal de salud existen jerarquías muy marcadas que propician y justifican el abuso de poder de unos a otros. Llama la atención cómo, en la formación del personal de salud, se rompen todas las reglas sobre igualdad, cordialidad y justicia.

Otro acto que atenta contra la ética y la justicia es la carga de trabajo. Los resultados muestran una disparidad abusiva en cuanto a su distribución. Los estudiantes ingresan al Internado para aprender, reforzar y consolidar sus conocimientos, no para ser fuerza de trabajo o para ser explotados. Los responsables de la atención, el cuidado del paciente y la demanda de atención son los médicos adscritos y el personal de base del hospital, no los internos. La cantidad no es el factor determinante para asegurar la calidad o el aprendizaje. Baste lo anterior para mostrar la clara negligencia y abuso por parte del personal y autoridades hospitalarias, y la clara explotación de los estudiantes, quienes no pueden oponerse a las demandas y órdenes de las autoridades.

\section{Alimentación}

En su artículo 7.7 la NOM estipula que los apoyos y ayudas mínimos que las instituciones de salud deben proporcionar a los alumnos incluyen beca, alimentos, uniformes y asistencia médicoquirúrgica. Al respecto, se encontró que los estudiantes no cuentan con el tiempo suficiente para su alimentación, porque las autoridades los interrumpen; eso explica por qué la mitad de la población de alumnos tuvo dos comidas o menos.

\section{Atención en salud}

El hospital es un centro para el cuidado, mejora y restablecimiento de la salud de cualquier persona. Se esperaría, siguiendo la lógica más básica y por sentido común, que entre el propio personal se 
fomentara y cuidara la salud. Cada unidad concentra a los "expertos" en la materia y su trabajo y funciones están dedicados en teoría a ese fin. No obstante, los resultados son preocupantes: nuevamente hay un vacío respecto de una cultura del cuidado y fomento de la salud.

La primera acción que realiza un médico a un paciente es la elaboración de su historia clínica. Ella muestra el estado actual de la persona y, con esos datos, se puede ver la evolución de su salud en el tiempo. Una vez más, contrario a la práctica común, a los estudiantes no se les genera un estudio ni un registro sobre su salud. Al estar en una unidad especializada sería lo mínimo que podrían recibir, además de realizarlo como una medida de salud preventiva. Sin embargo, a pesar de estar todo un año en el hospital, no se les hace ningún estudio, mucho menos un seguimiento o evaluación de su salud.

\section{Clases y conocimientos}

Aunque la mayoría respondió que pudo aplicar sus conocimientos previos, un porcentaje importante mencionó que el Internado no lo dotó de las competencias y habilidades necesarias para la atención del paciente. Otro dato que merece un análisis detallado es que los estudiantes consideraron que sus calificaciones no reflejaron su aprovechamiento.

Un factor destacable es que a más del $50 \%$ sus propios maestros no lo respetaron durante sus horarios de clase. O que los dejaran solos para irse a dormir, o atendiendo las guardias nocturnas o realizando procedimientos por primera vez sin instrucción. Ello es un atentado directo contra la norma y el propio mandato del internado, y contra las responsabilidades de los médicos adscritos.

Como resultado de lo anterior, solo el 37\% de los internos consideró que al docente le importó su aprovechamiento. En lo relacionado con las clases, casi la mitad de las veces quienes las dictaron fueron los internos. ¿¿Dónde está la asesoría, vigilancia y seguimiento de los adscritos?

Como se puede apreciar, el escenario es muy adverso. Poco respeto por sus horarios de aprendizaje, sensación de poco interés por el docente, inconformidad en la evaluación y menos asesoría de la esperada en comparación con la gran carga de trabajo asignada.

\section{Contacto con familiares y actividades recreativas}

Al buscar actividades que contrarrestaran las presiones y desgaste al que son sometidos, se encontró que no les queda tiempo para estar con sus seres queridos, familiares y amigos. También disminuyen mucho las actividades deportivas y recreativas. El riesgo al que están sometidos es muy alto y no se están desarrollando acciones para mejorar su salud y aprendizaje.

Mala alimentación, poco descanso, pocas horas de sueño, mucho trabajo, pocas horas de enseńanza, alta carga de trabajo, jornadas muy prolongadas, son las condiciones más frecuentes a lo largo de esta etapa.

\section{Experiencia positiva}

No obstante, curiosamente, para la gran mayoría de los internos el Internado fue una experiencia positiva para su desarrollo académico y formativo. Es muy contrastante encontrar esa respuesta después de los resultados anteriores. Es preocupante que, a pesar de haber sufrido tantas agresiones y violaciones a sus derechos, los estudiantes tengan una percepción positiva y pasen por alto todo lo anterior. Es grave que lo vean como algo positivo para su formación, pese a todas las violaciones de las que fueron víctimas.

El Internado es una etapa formativa no un campo de tortura. No se debe consentir violaciones a los derechos fundamentales de los alumnos, con acciones que atentan directamente contra la dignidad, con actos discriminatorios, con violencia y hostigamiento. Es triste que, todo un año de sus vidas, estén expuestos a estas prácticas en las unidades de mayor renombre y con los médicos más destacados del país.

Las autoridades médicas no pueden seguir hablando de bioética cuando todas estas malas prácticas siguen existiendo y son parte de la formación médica.

Los resultados de este estudio inicial hacen urgente que las autoridades tomen acciones para estu- 
diar estos resultados en profundidad y hacer un cambio en la deformación de la que son objeto los estudiantes.

Es muy importante que se realicen estudios similares en todos los países y a todo el personal de salud en sus diferentes etapas formativas. En el caso de los médicos, hacer estudios sobre el Internado, el Servicio Social y también en las Residencias.

Lo mismo para el caso de los estudiantes de enfermería y trabajo social. Finalmente, todos trabajan en el mismo lugar y son parte del mismo equipo. Todas las acciones les afectan igualmente y son parte del problema pero también de la solución. Por eso es importante hacer estudios de la formación y condiciones de vida de todos los actores comprometidos.
Se hace una atenta invitación para que se estudie el tema en todas las latitudes y se intente cambiar el modelo educativo, las condiciones y la cultura en la disciplina médica. Existen muchos estudios sobre el Síndrome de Burnout en internos y residentes de todas partes del mundo, y los resultados parecen confirmar una realidad compartida globalmente.

\section{Agradecimientos}

A la Dra. Janneth Carballido Vieyra, en su momento Coordinadora de Orientación Educativa de la ESM del IPN, por su valioso apoyo para la obtención de la información y su interés en la investigación.

\section{Referencias}

1. García Romero H, Limón Limón L. Bioética general. México: Ed. Trillas; 2009.

2. Fernández S. La violencia de género en las prácticas institucionales de salud: afectaciones del derecho a la salud y a las condiciones de trabajo en salud. Revista Gerenc. Polit. Salud, Colombia 2007; 6(12): 52-76.

3. Escuela Superior de Medicina. Programa Académico de Internado Médico. México: ESM; 2008.

4. Diario Oficial de la Federación. Norma Oficial Mexicana NOM-234-SSA1-2003. Utilización de campos clínicos para ciclos clínicos e internado de pregrado: 2003. Disponible en: http://www.salud.gob.mx/unidades/cdi/nom/234ssa103. html (Accesado el día 20 de noviembre 2012).

Recibido: 27 de septiembre de 2013

Aceptado: 3 de enero de 2014 